\title{
Ion Transport by Viscous Gas Flow through Capillaries
}

\author{
Baiwei Lin and Jan Sunner \\ Department of Chemistry, Montana State University, Bozeman, Montana, USA
}

\begin{abstract}
The effects of a number of experimental parameters on the efficiency of ion transport by viscous gas flow through narrow capillaries have been studied. Both electrospray and corona ion sources were used. The experimental data are consistent with ions loss to the walls of the capillary, which initially is caused mainly by space-charge expansion, but later is caused by diffusion. These processes can result in severe discrimination against low mass ions. The extent of ion loss may be calculated by using a simple model for radial diffusional loss in long cylinders, with an exponential decay of the ion density along the transport capillary. However, such a simple model underestimates ion loss by ignoring the effects of space-charge, turbulent flow, and rapid decay of higher radial diffusion modes (enhanced loss of ions that enter the capillary close to the wall). In contrast, Monte Carlo simulations showed that the effect of the parabolic velocity profile, under laminar flow conditions, is to increase the transmitted ion current, sometimes by several orders of magnitude, relative to the predictions of the simple diffusion model. After considering all these factors, the transmitted current from a corona was well reproduced by using mobility values for ions formed in such discharges. However, the measured transmitted current from at electrospray source was much too high. To explain this, it was necessary to assume that about $2 \%$ of the electrospray current is carried by aerosol particles with radii in the 10-25- $\AA$ range. Finally, it is argued that in glass capillaries wall charging may explain why the transmitted ion current is observed to be very similar to that in metal capillaries. (J Am Soc Mass Spectrom 1994, 5, 873-885)
\end{abstract}

$\mathrm{T}$ The development of the electrospray (ES) ionization method $[1,2]$ has had a tremendous impact on mass spectrometry. In contrast to electron ionization, chemical ionization, fast atom bombardment, and so forth, the ES source operates at atmospheric pressure. This means that the analyte ions must be transferred from a 1-atm environment into the vacuum of the mass spectrometer while the neutrals are pumped away. This is not a new problem in mass spectrometry. Atmospheric pressure ionization (API) mass spectrometry, which uses a corona discharge or a $\beta$-emitter such as ${ }^{63} \mathrm{Ni}$, has a long history [3].

Thus, Horning et al. [4] introduced API in 1973. There are two basic designs for interfacing an API source with a mass spectrometer. In one, the ions pass directly from atmospheric pressure into the vacuum chamber through a single pinhole orifice [5]. Here, the gas flow through a $\sim 100-\mu$ m-diameter orifice is pumped by a very efficient cryopump on the vacuum side. Electrical fields are used to extract the ions from the gas jet. The extensive cooling in the supersonic jet may result in unwanted cluster ions, particularly with water molecules. Therefore, a dry nitrogen interface

Address reprint requests to Professor Jan Surner, Department of Chemistry, Montana State University, Bozeman, MT 59717. gas is introduced at the high pressure side of the orifice [5]. This API design is presently used in some electrospray sources.

In a second API design, the pressure drop is achieved in two stages. The ions first pass through an orifice and enter a chamber with a pressure in the torr range. This chamber is usually pumped by a mechanical rough pump. The ions are then sampled into the mass spectrometer through a conical skimmer [6]. This API design is very common for electrospray interfaces. Also with this design it can be beneficial to force the ions to drift through a drying gas at atmospheric pressure prior to entering the intermediate pressure chamber [7]. The ions may be heated by applying a high electric field, either in the intermediate pressure chamber or in the supersonic jet $[8,9]$. This results in declustering or even fragmentation. Such heating can frequently substitute for a dry curtain gas.

An elegant variation of the API interface was developed by Whitehouse et al. [7] in 1985. These authors connected the atmospheric pressure chamber with the intermediate pumping chamber by means of a $0.2 \times$ 60 -mm glass capillary. The gas throughput was close to that of a $100-\mu \mathrm{m}$ orifice and equally good mass spectra were obtained [7]. There are clear advantages with the capillary interface. First, the design of the API 
source is often simplified as the distance between the electrospray and the intermediate pressure chamber is increased. Second, the viscous flow in the glass capillary allows for the ions to be transported against an electrical field. Thus, it was reported [7] that the ions could be "pumped" through a potential difference of more than $15 \mathrm{kV}$. Third, by heating the capillary, desolvation of electrospray droplets can be accomplished without a curtain gas [10]: By heating the capillary to higher temperatures, the ions even can be induced to fragment [11].

For mass spectrometrists, used to working with low pressure ion sources, ion transport through a narrow capillary seemed a surprising and strange phenomenon. It is tempting to assume that with a capillary radius as small as $0.1 \mathrm{~mm}$, very few ions would escape colliding with the capillary walls. However, experiments proved otherwise and the lore among electrospray practitioners has been that ions would pass through even very long capillaries. There remains much uncertainty as to the mechanism of ion transport through capillary tubes at high pressure. There are several possibilities. For example, with electrospray ionization charged droplets in addition to gas-phase ions may pass through the capillary. With glass capillaries the insulating walls may become charged as the first ions hit the wall. It then can be argued that the repulsion between like charges must, at some point, prevent further charging of the wall and that ions that enter the capillary have no choice but to pass through the entire length of the capillary. A charged wall can indeed have some surprising effects on moving ions [12]. Such ideas provided the impetus for the present work.

In this article, we present an experimental study of ion transport by viscous gas flow through narrow capillaries. The effects of a number of experimental parameters, including capillary dimensions and material, gas throughput, ionization method (electrospray and corona), and temperature, on ion throughput have been studied.

\section{Experimental}

The experimental setup is shown in Figure 1. The main parts of the setup are electrospray capillary, counterelectrode, transport capillary, and ion collection electrode assembly. The cylindrical ion source has an inner diameter of 3.5 in. and a length of 4.5 in. The ES capillary was mounted along the ion source center axis. The capillary-to-counterelectrode distance was 2 $\mathrm{cm}$ unless otherwise specified; it could be varied by moving the capillary longitudinally by means of a screw mechanism. A motor-driven syringe pump (Sage model 355, Orion Research, Inc., Boston, MA) was used to force $4.4 \mu \mathrm{L} / \mathrm{min}$ (except where noted) of a $1.0 \times 10^{-4}-\mathrm{M}$ sodium acetate solution in $50 \mathrm{vol} \%$ methanol in water through the stainless steel electrospray capillary (o.d. $1.0 \mathrm{~mm}$ and i.d. $0.5 \mathrm{~mm}$ ). About 4-6 kV was applied to the spray needle by a high voltage supply (Glassman PS/EH 60R01.5, Whitehouse Station, NJ) while the counterelectrode was grounded. The electrospray current $I_{\text {es }}$ was monitored with a digital voltmeter (Keithley 169, Cleveland, $\mathrm{OH}$ ) in series with the high voltage supply. A positive voltage of $10-50 \mathrm{~V}$ was applied to the wall of the ES ion source (but had a negligible effect on the transmitted current). The ion source was wrapped with heating tape and the source temperature was maintained at 35 ${ }^{\circ} \mathrm{C}$, except where noted. The source atmosphere was room air. When dry nitrogen was used, no differences relative to air were observed. In the corona source, a needle was substituted for the ES capillary.

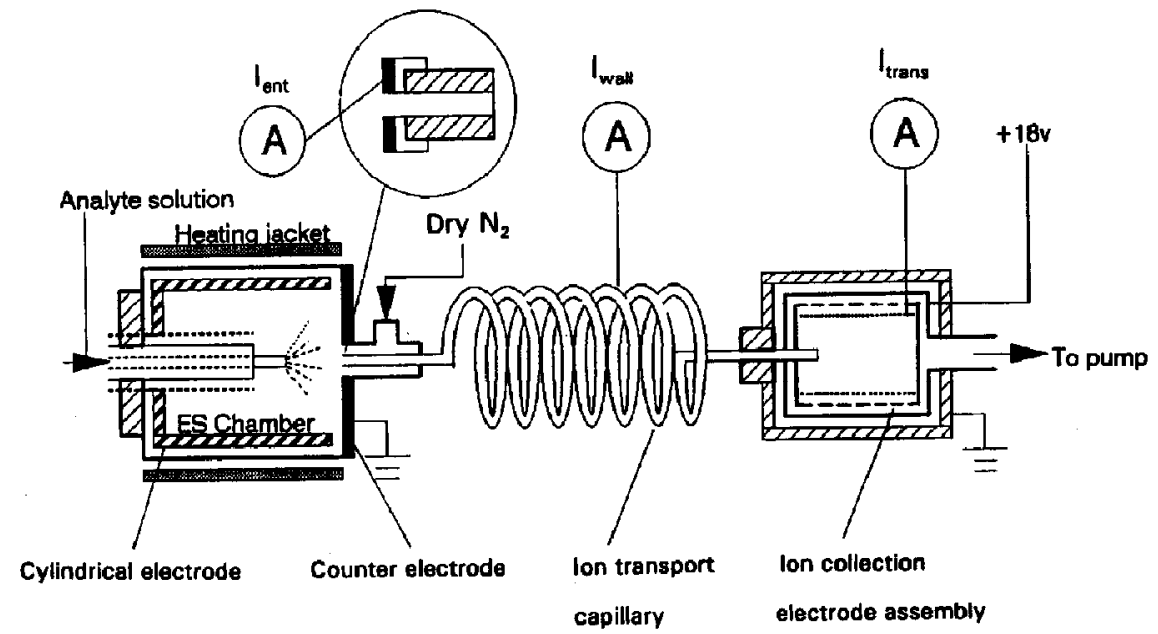

Figure 1. Experimental setup for ion transmission studies. Currents were measured at points labeled A. 
Ion transport capillaries of stainless steel, glass, and Teflon ${ }^{(8)}$ were used. The inner diameter of the capillaries varied from 0.97 to $3.8 \mathrm{~mm}$ and the lengths from 25 $\mathrm{cm}$ to $15 \mathrm{~m}$. A stainless steel washer-shaped "entrance" electrode was mounted on the front butt end of the transport capillary and isolated from the capillary with a thin ceramic spacer (see insert to Figure 1). The inner diameter of the entrance electrode was nearly the same as that of the transport capillary so as not to restrict the air flow into the capillary. The outer diameter was 6 $\mathrm{mm}$. The entrance current $I_{\mathrm{ent}}$ owing to ions hitting the entrance electrode was measured by a homemade current amplifier, built from operational amplifiers. When metal transport capillaries were used, the current owing to ions hitting the inner wall $I_{\text {wall }}$ was measured with a picoammeter (Keithley 485).

The collection of ions at the end of the transport capillary had to be highly efficient. This presented a problem because a high gas throughput at a relatively high pressure tends to sweep ions out of the collection chamber. In the final design, the ion collector consisted of two concentric cylindrical electrodes. The inner cylinder was made from a brass screen (60 mesh) and the outer cylinder was made from a copper cylinder through which a number of narrow holes had been drilled for pumping. This dual electrode was connected through a double-shielded cable to a picoammeter (Keithley 617). The electrode assembly was housed in a copper vacuum jacket evacuated by a 2.1 $\mathrm{ft}^{3} /$ min rough pump (Welch, Skokie, IL). Two 9-V batteries were used to apply a repeller voltage $(+18 \mathrm{~V})$ to the outer vacuum housing relative to the dual collection electrode. The measured transmitted current $I_{\text {trans }}$ was the same whether this repeller voltage was +9 or $+18 \mathrm{~V}$, which provides evidence that the ion collection was highly efficient. The ion transport capillary gas throughput was controlled by a valve in the vacuum line between the ion collection assembly and the vacuum pump and measured with a variable area flowmeter connected over a filter to the pump exhaust. The pressure at the ion collection assembly varied inversely with the capillary air flow from a few torr to close to atmospheric pressure.

The entrance current $I_{\text {ent }}$ was sensitive to small variations in the assembly of the ion source and could vary by about a factor of 2 between different experiments. However, the sum of $I_{\text {wall }}$ and $I_{\text {trans }}$ varied much less, when compared at the same gas throughput. All current measurements represent steady state values; we were not equipped to study fast current changes. The four current signals $\left(I_{\mathrm{es}}, I_{\mathrm{ent}}, I_{\text {wall }}\right.$, and $I_{\text {trans }}$ ) were monitored, through the analog signal outputs from the amplifiers and current meters, by a computer interface (Montana Laboratory Interface, Bozeman, MT) and stored on a 8088-based personal computer. The background currents were negligible unless atherwise noted. A spreadsheet program was used for data storage, manipulation, plotting, and transport modeling. The Monte Carlo program for sim-
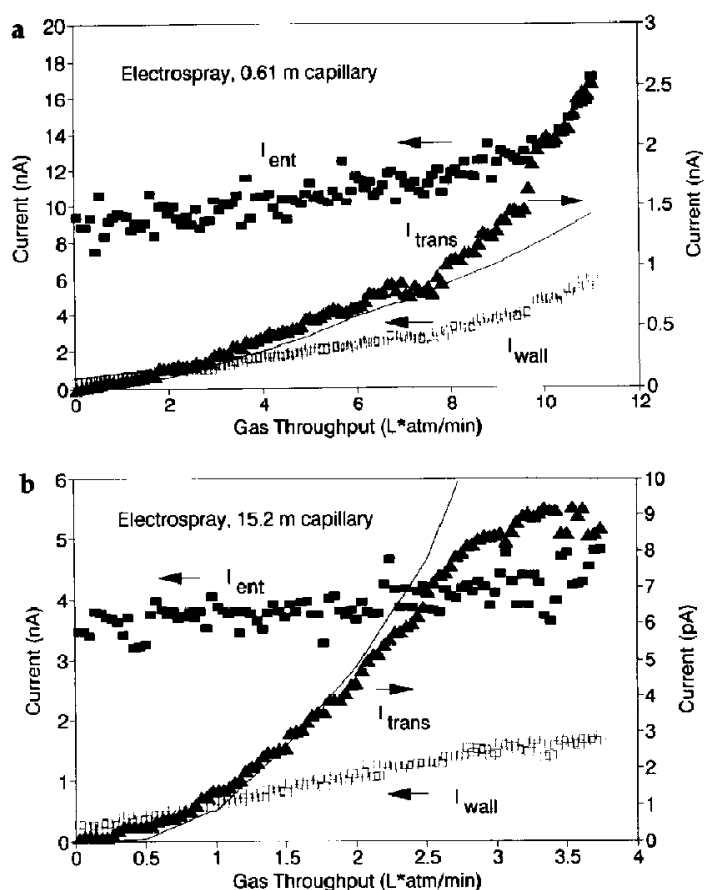

Figure 2. (a) Entrance $\left(I_{\mathrm{e} n \mathrm{l}}\right)$, wall ( $\left.I_{\text {wall }}\right)$, and transmitted $\left(I_{\text {trans }}\right)$ currents as a function of gas throughput for an $2.1-\mathrm{mm}-\mathrm{i} . \mathrm{d}$. and $0.61-\mathrm{m}$-long stainless steel capillary and electrospray ionization. Other conditions were as described in the experimental section. The solid line shows $I_{\text {trans }}$ calculated with the full diffusion/ space-charge model and the diffusion constants in Table 1. (b) Same as (a), but the length of the transport capillary was $15.2 \mathrm{~m}$.

ulating the ion diffusion in the transport capillaries was written in QBASIC.

\section{Results}

\section{Metal Capillaries}

The electrospray, entrance, wall, and transmitted currents were measured with the setup shown in Figure 1 for a series of $2.1-\mathrm{mm}$-i.d. stainless steel capillaries with lengths from 0.61 to $15.2 \mathrm{~m}$. Figure $2 \mathrm{a}$ shows the currents for the $0.61-\mathrm{m}$ capillary as a function of the gas throughput from 0 to $11 \mathrm{~L} \cdot \mathrm{atm} / \mathrm{min}$. The entrance current of about $10 \mathrm{nA}$ constituted about $5 \%$ of the total electrospray current of $0.2 \mu \mathrm{A}$. The moderate increase of the entrance current from 9 to $16.5 \mathrm{nA}$ with increasing throughput is probably due to higher ion density gas volumes being swept, by viscous gas flow, from the proximity of the ES needle into the region close to the entrance electrode. Further, it is seen in Figure $2 \mathrm{a}$ that the capillary wall current is positive $(0.2$ nA) even at zero gas flow. This is due to ions that diffuse or drift past the entrance electrode into the capillary. As the gas flow increases, the wall current is nearly proportional to the gas throughput and reaches a maximum of $6 \mathrm{nA}$. At the same time, the transmi'ted 
current increases from 0 to $2.5 \mathrm{nA}$. The ratio

$$
T_{\text {eff }}=\frac{I_{\text {trans }}}{I_{\text {wall }}+I_{\text {trans }}}
$$

can be used as a figure of merit for the efficiency of ion transmission through the capillary. This ratio increases from 0 to $29 \%$ with increasing throughput.

Figure $2 b$ shows results analogous to Figure $2 a$ except that the transport capillary was much longer, $15.2 \mathrm{~m}$. The two graphs look qualitatively very similar (although they do reflect the large run-to-run variance in $I_{\text {ent }}$. As was the case for the shorter capillary, the wall current $I_{\text {wall }}$ increases nearly linearly and the transmitted current $I_{\text {trans }}$ more rapidly with increasing gas throughput. A minor difference is observed at high gas throughput where $I_{\text {trans }}$ levels off in Figure $2 \mathrm{~b}$. This behavior was observed for capillaries longer than about $2 \mathrm{~m}$. The maximum transmission efficiency through the $15.2-\mathrm{m}$ capillary was only $0.5 \%$, compared to $21 \%$ for the $0.61-\mathrm{m}$ capillary at the same gas throughput $(3.7 \mathrm{~L} \cdot \mathrm{atm} / \mathrm{min})$.

Electrospray current measurements such as those shown in Figure 2a and $b$ were plotted versus several different parameters to elucidate what experimental factors influence the ion transmission efficiency. Of particular interest is the plot in Figure 3, which shows the transmitted current for several 2.1-mm-i.d. metal capillaries with lengths between 0.61 and $15.2 \mathrm{~m}$ and a gas throughput between 1 and $5 \mathrm{~L} \cdot \mathrm{atm} / \mathrm{min}$. In this graph, the transmitted current is plotted versus the 1-atm (ion) residence time defined by

$$
t_{\text {res }}^{0}=\frac{V_{\text {cap }}}{F_{1 \text { atm }}}=\frac{V_{\text {cap }} \times(1 \mathrm{~atm})}{Q}
$$

where $V_{\text {cap }}$ is the capillary volume, $F_{1}$ atm is the gas volume flow rate at $1 \mathrm{~atm}$, and $Q$ is the gas throughput. If there was no pressure loss through the capil-

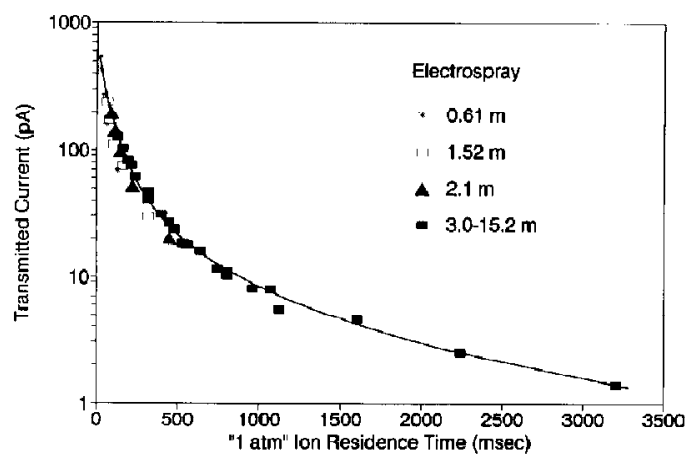

Figure 3. The ion current ( $I_{\text {Irans }}$ ) from an electrospray source transmitted through $2.1-\mathrm{mm}$-i.d. stainless steel capillaries as a function of the 1-atm ion residence time (eq 2) of the ions in the capillary. The capillary lengths were $0.61(*), 1.52(\square)$, and $2.1 \mathrm{~m}$ ( 4 ), and several lengths from 3.0 to $15.2 \mathrm{~m}$ ( $\square$ ). The gas throughput varied from 1.0 to $5.0 \mathrm{~L} \cdot \mathrm{atm} / \mathrm{min}$. The solid line is a simple fit through the experimental points.

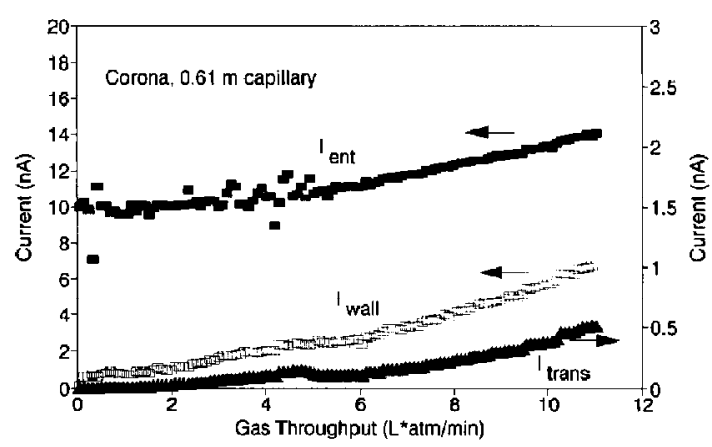

Figure 4. Same as Figure $2 a$, except that a corona ion source was used instead of an electrospray ion source.

lary, $t_{\text {res }}^{0}$ would be the actual residence time for the ions in the capillary. However, in reality the pressure drops, the linear gas velocity increases, and the actual residence time is shortened. It is seen in Figure 3 that the transmitted current data fall nearly on a single line, even though a wide range of experimental conditions was used. However, there is some scatter, particularly at short ion residence times. Here $I_{\text {trans }}$ is smaller by a factor of about 2 for the shorter capillaries.

With the electrospray ion source it is always possible that charged particles or droplets are responsible for part of the transmitted current. Therefore, a room air corona discharge ion source was used for comparison. The ions sampled from such a source are either hydronium ion hydrates, $\mathrm{H}_{3} \mathrm{O}^{+}\left(\mathrm{H}_{2} \mathrm{O}\right)_{n}$, or hydrates of protonated high proton affinity impurities, $\mathrm{MH}^{+}\left(\mathrm{H}_{2} \mathrm{O}\right)_{n}$ [13]. Figure 4 shows the result for the 2.1-mm-i.d. $\times 0.61-\mathrm{m}$-long capillary. The major difference relative to the electrospray result in Figure 2a (with the same capillary) is that the transmitted corona current is considerably smaller. For example, the maxi-

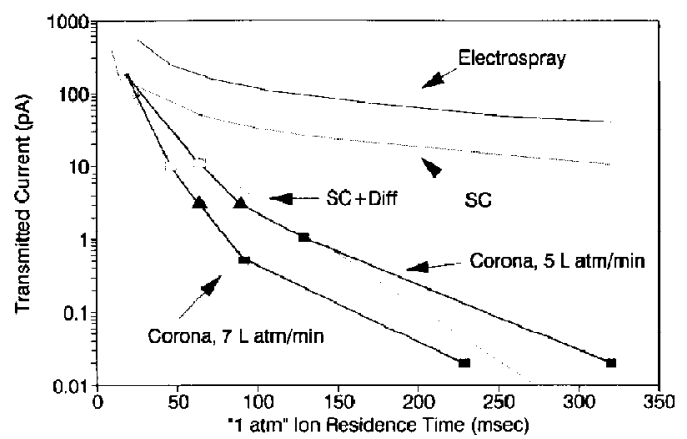

Figure 5. The ion current $\left(I_{\text {trans }}\right)$ from a corona source transmitted through 2.1-mm-i.d. stainless steel capillaries as a function of the 1-atm ion residence time (eq 2). Capillary lengths varied from 0.61 to $7.6 \mathrm{~m}$. Data for gas throughput of $5(\square)$ and $7(\square)$ $\mathrm{L} \cdot \mathrm{atm} / \mathrm{min}$ are shown. The point shapes represent different length capillaries; see Figure 3. For a comparison, the line marked "Electrospray" shows the electrospray current from Figure 3 at corresponding ion residence times. The line " $\mathrm{SC}$ " shows $l_{\text {trans }}$ calculated assuming that space-charge induced ion loss is the only loss mechanism. The line marked "SC + Diff" shows $I_{\text {trans }}$ calculated using the full diffusion/space-charge model and the diffusion constants in Table 1 . 
mum transmission efficiency at $11 \mathrm{~L} \cdot \mathrm{atm} / \mathrm{min}$ is $\sim 7 \%$ for the corona versus $29 \%$ for the electrospray. As we will see, this difference becomes much larger for long capillaries.

The transmitted corona current was measured for several capillaries with lengths from 0.61 to $7.6 \mathrm{~m}$; see experimental points in Figure 5. The comparable electrospray result from. Figure 3 is shown by the line marked "Electrospray." It is seen that the transmitted current decreases much faster with ion residence time for the corona than for the electrospray. Also, there is a considerable loss of transmitted corona current as the gas throughput increases from 5 to $7 \mathrm{~L}$ - atm/ $\mathrm{min}$ at the same $t_{\mathrm{res}}^{\mathrm{O}}$.

\section{Glass Capillaries}

It was more difficult to obtain stable and reproducible currents with glass than with metal capillaries. Teflon capillaries were even harder to work with. Presumably this is because of charging of the glass and Teflon surfaces. Furthermore, there is no wall current to measure and only the electrospray, entrance, and transmitted currents could be recorded. The transmission efficiency according to eq 1 thus could not be calculated. Instead, we will use the ratio of $I_{\text {trars }}$ to $I_{\text {ent }}$ as a measure of the ion transmission efficiency for glass capillaries. Only relative values of this ratio will be discussed; the absolute values depend on the size of the entrance electrode.

Glass and metal capillaries were compared under identical conditions. For example, in one series of experiments, capillaries with lengths from 0.30 to $3.0 \mathrm{~m}$ were used (i.d. $2.0 \mathrm{~mm}$ ). The dependence on gas throughput was qualitatively very similar to that seen for metal capillaries in Figure 2a. However, with ion residence times from 10 to $140 \mathrm{~ms}$, it was found that the transmitted current with glass consistently was about a factor of 2 lower than for the metal capillary. In no case did we observe a higher transmitted current for a glass (or Teflon) than for a metal capillary of same dimensions.

The transmitted current through a particular capillary depends not only on gas throughput, but also on the experimental conditions in the electrospray source. We studied the effects of a large number of experimental parameters with the glass capillaries. Figure 6 shows a typical result. In this experiment, the distance between the electrospray needle tip and the transport capillary entrance was increased from 5 to $40 \mathrm{~mm}$. It is seen that whereas the entrance current $I_{\text {ent }}$ decreased by a factor of $\sim 50$, the transmitted current $I_{\text {trans }}$ only decreased by a factor of 5 . Thus, the ratio of $I_{\text {trans }}$ to $I_{\text {ent }}$ (and thus the ion transmission efficiency) increased by a factor of about 10. An increase in ion transmission efficiency with decreasing entrance current, and vice versa, was observed not only when the capillary distance was varied but also when the ES voltage or solution flow rate were varied (results not shown). A notable exception was the effect of temperature.

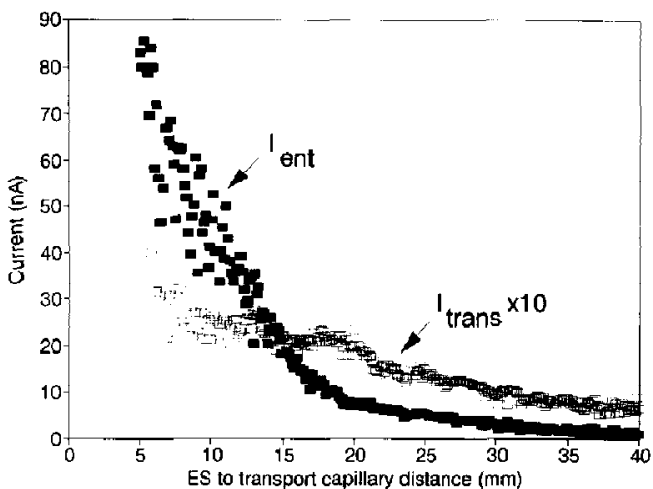

Figure 6. Entrance $\left(I_{\text {ent }}\right)$ and transmitted $\left(I_{\text {trans }}\right)$ currents through a glass capillary as a function of the distance from the electrospray capillary tip to the transport capillary entrance. Capillary length $0.25 \mathrm{~m}$ and diameter $1.47 \mathrm{~mm}$.

Figure 7 shows that both the entrance current and the $I_{\text {trans }}$ to $I_{\text {ent }}$ ratio increased with increasing ion source temperature.

The transmitted current was measured for a number of glass capillaries with different dimensions. Figure 8 shows the results for capillaries of the same lengths but with different diameters. In Figure $8 \mathbf{a}_{r} I_{\text {trans }}$ is plotted versus $t_{\text {ress }}^{0}$, that is, the 1-atm ion residence time from eq 2. After the first few milliseconds, the current decays exponentially with $t_{\text {res, }}^{\prime \prime}$ as seen from the straight lines in this semilogarithmic graph. Also, far fewer ions are transmitted in the narrower capillaries at the same $t_{\mathrm{res}}^{0}$. In Figure $8 \mathrm{~b}$, the same data are plotted versus gas throughput. It is remarkable that all the data now fall nearly on the same line even though they were obtained with different inner diameter capillaries.

\section{Discussion}

The main problem addressed by this work relates to the nature of ion transport and ion loss as the ions are

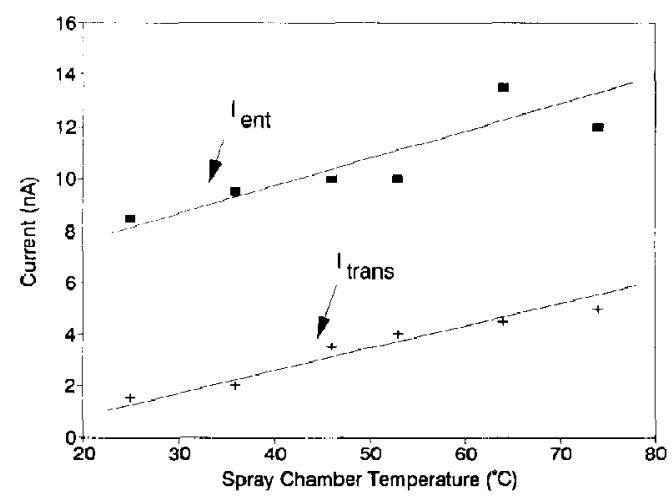

Figure 7. Entrance $\left(I_{e n t}\right)$ and transmitted $\left(I_{\text {trans }}\right)$ currents through a glass capillary, length $0.25 \mathrm{~m}$ and diameter $1.47 \mathrm{~mm}$, as a function of the temperature of the electrospray ion source. 

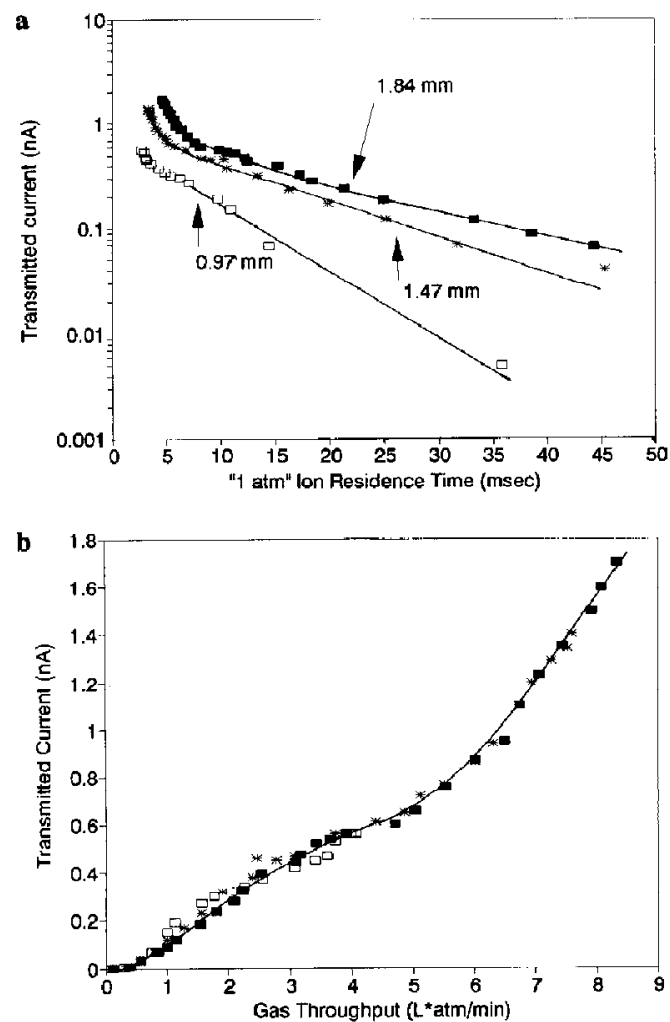

Figure 8. (a) Electrospray ion current transmitted ( $\left(I_{\text {trans }}\right)$ through three glass capillaries, all $0.25 \mathrm{~m}$ long, with the different inner diameters given in the figure. (b) Same transmitted ion current data as in (a), but plotted versus the gas throughput

transported by viscous gas flow through narrow capillaries. The conventional expectation must be that ions are transported from the center of the capillary toward the wall by the well known processes of diffusion and ion drift in the electric field set up by the space-charge. This is certainly the case for metal capillaries where the ions should be discharged when they hit the wall and cause a wall current to flow. The mathematical treatments of the space-charge and diffusion ion loss processes will first be developed. The experimental results are then compared with the predictions of the equations for the two ion loss processes separately to see what process(es) may be important. A calculation that includes both processes is then presented and applied to both the corona and the electrospray results. The ion transport through glass capillaries is discussed, and finally the implications of the results for electrospray are briefly considered.

\section{Space-Charge and Diffusion in Narrow Metal Capillaries}

The mathematical treatment of space-charge-driven ion loss in the transport capillaries will first be devel- oped, ignoring for now diffusional loss. The electrospray ion source is a space-charge-dominated unipolar ion source [14]. This means that the charge density $\rho(t)$ in an "ion cloud" that drifts from the capillary tip to the counterelectrode is given by the unipolar formula $[15,16]$,

$$
\frac{1}{\rho(t)}=\frac{1}{\rho(t=0)}+\frac{\kappa}{\epsilon} t
$$

where $t$ is time, $\rho(t=0)$ is the charge density at time zero, $\kappa$ is the ion mobility, and $\epsilon$ is the electric permittivity. (Throughout this paper, we have used the vacuum permittivity for $\epsilon$ ) Time zero may be arbitrarily chosen, but a natural choice is the time of initial formation of gas-phase ions. One then obtains the ion density at the counterelectrode $\rho\left(t_{d}\right)$, at $t=t_{d}$, where $t_{d}$ is the ion drift time from capillary to counterelectrode. Fortunately, for typical (long) drift times, $p\left(t_{d}\right)$ is only a very weak function of $\rho(t=0)$ [14]. As will be discussed later, the space-charge calculation in electrospray ion sources is complicated by the fact that the charges have a distribution of mobilities.

When the unipolar formula is applied to ion transport through the capillary, time zero refers to the time when a "charge cloud" just enters the capillary. The charge density at the capillary exit (assuming that diffusion is negligible) is then obtained by setting the time in eq 3 to $t_{\text {res }}$, that is, the ion residence time in the capillary. The charge density at the entrance of the transport capillary is presumably nearly the same as the charge density at the electrospray counterelectrode. This charge density, which will be denoted $\rho_{\text {cnt }}$ can be calculated from the experimental data by,

$$
\rho_{\text {cnt }}=\frac{I_{\text {wall }}+I_{\text {trans }}}{F_{1 \text { atm }}}
$$

where $F_{1 \mathrm{~atm}}$ is the volume flow rate (at $1 \mathrm{~atm}$ ). In the present experiments $\rho_{\mathrm{ent}}$ was consistently found to be $2-2.5 \times 10^{8}$ ions $/ \mathrm{cm}^{3}$ at a $20-\mathrm{mm}$ electrospray emitter to (metal) transport capillary distance.

A problem with applying the unipolar formula to capillary ion transport is that the pressure decreases substantially along the capillary at a high gas throughput. However, the gas velocity is inversely proportional to the pressure. Therefore, the ion residence time in a segment of the capillary is proportional to the pressure within the capillary. Now, the mobility $\kappa$ is inversely proportional to the pressure [17]. Thus, according to eq 3 , the increase in the mobility constant will be canceled by a decrease in the (ion residence) time $t$. Thus for applications of the unipolar formula, the pressure drop in the capillary can be ignored and the 1-atm ion residence time $t_{\text {res }}^{0}$ can be used together with the mobility constant at 1 atm.

We now turn to the treatment of ion loss by diffusion, assuming for now that space-charge-driven ion 
loss is negligible. Fick's law of diffusion,

$$
\bar{j}=-D \bar{\nabla} n
$$

states that the ion flux density $\bar{J}$, (at any given point in time and space), is proportional to the diffusion constant $D$ and to the gradient of the number density of the ions $n$. Equation 5, together with the equation of continuity, is used to derive the time-dependent diffusion equation [17]

$$
\left(\frac{\partial n}{\partial t}\right)=\bar{\nabla} \cdot(D \bar{\nabla} n)
$$

The radial diffusion of ions in a cylinder of radius $r_{0}$ with zero ion density at the wall has a known solution [17]. For an infinitely long cylinder, the charge density as a function of radius $r$ and time $t$ is given by

$$
\rho(r, t)=\sum_{i=1}^{\infty}\left[G_{i} J_{0}\left(\alpha_{i} r\right) \exp \left(-\frac{t}{\tau_{i}}\right)\right]
$$

where $G_{i}$ is a constant, $J_{0}$ is a Bessel function of order zero, $\alpha_{i} r_{b}$ is the $i$ th root of $J_{0}$, and $\tau_{i}$ are time decay constants. The important conclusion from eq 7 is that the ion density in the cylinder can be seen as a sum of radial diffusion modes each with a different decay time $\tau_{i}$. For the fundamental diffusion mode $(i=1)$,

$$
\begin{gathered}
\tau_{1}=\left(\frac{1}{D}\right)\left(\frac{r_{0}}{2.405}\right)^{2} \\
\rho(r, t)=\rho_{0}(r) \exp \left(-\frac{t}{\tau_{1}}\right)
\end{gathered}
$$

The higher diffusion modes decay faster. For example, the lifetime of the second diffusion mode is 5.3 times shorter than that of the first. This means that if the ion density initially is constant over the capillary cross section (as in ion source gas when it enters the capillary), the ion loss at first will be more rapid than predicted by eq 8 . It is easy to understand why. Those ions that are initially close to the wall have a high chance of hitting the wall within a short time. As they do, the radial ion density distribution changes to that of the fundamental diffusion mode, where the ion density is at a maximum in the center of the cylinder and gradually decreases to zero at the wall. Once this diffusion mode is established, the rate of ion loss should be that predicted by eq 8 . Such behavior in the experimental data is seen in Figure 8a.

The application of eqs 7 and 8 to ion diffusion, not in a stationary gas, but in a gas flowing through a capillary, will now be discussed. The flowing gas introduces additional difficulties: the pressure drop through the capillary, the possibility of turbulent flow, and the parabolic flow profile under laminar flow conditions.

As was shown above, in applications of the unipolar formula (eq 3) the pressure drop through the capillary could be ignored because the increase in the mobility constant $\kappa$ with decreasing pressure was canceled by a proportional decrease in the ion residence time. What about the diffusion equations? The diffusion constant $D$ is inversely proportional to pressure [17]. Equation $8 \mathrm{a}$ then shows that $\tau_{1}$ is proportional to the pressure. However, as already shown in the discussion of the unipolar formula, the ion residence time (in any section of the transport capillary) is proportional to the pressure. Thus, in eqs 7 and $8 \mathrm{~b}$, the increase in the diffusion constant with decreasing pressure is canceled by a proportional decrease in the ion residence time. Thus, when eqs 7 and 8 are used, we can use diffusion constants at $1 \mathrm{~atm}$ and the $\mathbf{1 - a t m}$ ion residence time.

It is expected that the extent of ion loss in a transport capillary will depend on whether the flow is turbulent and laminar. First, it should be noted that for the pressure range encountered inside the capillaries, the mean free path is much smaller than the dimensions of the capillaries used here. Therefore, the flow is viscous. Whether the flow is turbulent or laminar depends on the Reynolds' number,

$$
\operatorname{Re}=\frac{v_{\text {ave }} \rho_{\mathrm{g}} d}{\eta}
$$

where $v_{\text {ave }}$ is the average flow velocity, $\rho_{\mathrm{g}}$ is the density of the gas, $d$ is the inner diameter of the capillary, and $\eta$ is the viscosity of the gas. [Note that Re sometimes is defined by using the radius and not the diameter in eq 9. Also, because $\eta$ is (nearly) independent of $P, \rho_{\mathrm{g}}$ is proportional to $P$, and $v_{\text {ave }}$ is inversely proportional to $P$, Re is independent of $P$ and thus constant throughout a capillary.] The flow through a capillary tube is laminar for Reynolds numbers below 2100 and turbulent for Reynolds numbers above 4000 [18], In the transition region $\mathrm{Re}=$ 2100-4000, the flow may be either laminar or turbulent. In addition, there is a tendency for the flow to be turbulent close to the capillary entrance, even at Reynolds' numbers below 2100 [18]. For air ( $\eta=1.8 \times$ $10^{-5} \mathrm{P}$ and $\rho=1.1 \mathrm{~kg} / \mathrm{m}^{3}$ ) flowing through the 2.1 mm-i.d. capillary, the flow should be laminar for $Q<$ $3.2 \mathrm{~L} \cdot \mathrm{atm} / \mathrm{min}$ and turbulent for $Q>6 \mathrm{~L} \cdot \mathrm{atm} / \mathrm{min}$. Thus, we expect laminar flow for the 15.2-m capillary in Figure $2 \mathrm{~b}$ but turbulent flow for the $0.61-\mathrm{m}$ capillary in Figure 2a when the gas throughput is larger than 3-6 L $\cdot \mathrm{atm} / \mathrm{min}$.

The analysis of ion diffusion loss in narrow capillaries will be developed here for laminar flow only. One might expect that turbulent flow should significantly increase the rate of ion loss to the walls. However, as we will see, the laminar flow treatment will give an acceptable estimate of the extent of ion loss. For this reason, but also because of the complexity of the physics, we did not try to describe ion loss under turbulent flow conditions. Equations 7 and 8 would be 
directly applicable to the radial diffusion of ions in a gas that flows through a narrow capillary (under laminar flow conditions) if the gas velocity were the same throughout the capillary cross section ("plug" flow). This of course is not the case; the gas velocity at the wall is zero because of viscous drag and increases toward the capillary center. The velocity profile $v(r)$ is known to be parabolic [18]:

$$
v(r)=2 v_{\text {ave }}\left(1-\left(\frac{r}{r_{0}}\right)^{2}\right)
$$

where $r$ is the distance from the center of the capillary and $r_{0}$ is the capillary radius. The parabolic flow profile means that eqs 7 and 8 are not applicable directly to gas flow through a capillary. The problem previously has been studied in connection with selected ion flowing afterglow experiments [19]. Here we have used a Monte Carlo simulation to solve the problem; see the Appendix. The conclusion is that the parabolic velocity profile can enhance ion transmission through long capillaries by orders of magnitude. Fortunately, the effect can be approximated simply by dividing the diffusion constant in eq 8 by a factor of about 1.6 as seen in the Appendix. The parabolic flow profile also should affect the space-charge-induced ion loss; however, because space-charge is only important close to the capillary entrance (see below) where the gas flow is very complicated, we did not consider it worthwhile to include this effect in our calculations.

\section{Corona Results}

Comparison of the electrospray and corona results in Figures 2 and 4 shows that the ion transmission efficiency was considerably lower for the corona ions. Furthermore, the comparison in Figure 5 showed that the corona-transmitted current decayed much faster with increasing ion residence time. Can these results be explained as a consequence of space-charge-driven or diffusional loss?

The $\mathrm{H}_{3} \mathrm{O}^{+}\left(\mathrm{H}_{2} \mathrm{O}\right)_{n}$ ions produced in a corona discharge in ambient air have 1-atm mobilities in the $2.3 \pm 0.4-\mathrm{cm}^{2} / \mathrm{V} \cdot \mathrm{s}$ range [20]. (Water clusters of protonated low molecular weight impurities would have similar mobilities.) Furthermore, the ion density at the capillary inlet $\rho_{0}$ in these corona experiments was about $2 \times 10^{8}$ ions $/ \mathrm{cm}^{3}$, calculated from eq 4 . At 5 $\mathrm{L} \cdot \mathrm{atm} / \mathrm{min}$, this corresponds to a current of $2.7 \mathrm{nA}$. With these data, the transmitted current predicted by the unipolar formula (eq 3) was calculated from

$$
I_{\text {trans }}=F_{1 \text { atm }} \times \rho\left(t_{\text {res }}^{0}\right)
$$

where the 1-atm residence time and flow rates were used. The result is shown by the line marked " $\mathrm{SC}$ " in Figure 5. It is seen that the predicted decay of $I_{\text {lrans }}$ is much too slow, and no reasonable changes in the calculation can change this result. This shows that space-charge-driven is important, but only during the initial $25 \mathrm{~ms}$. Indeed, by equating the diffusional charge loss $(\partial \rho / \partial t)$ (derived from eq 8 ) with the space-charge loss $(\partial \rho / \partial t)$ (derived from eq 3 ) and substituting Einstein's relation

$$
\kappa=\frac{z e D}{k T}
$$

where $z$ is the number of charges on each ion, $e$ is the elementary charge, $k$ is Boltzmann's constant, and $T$ is the absolute temperature, one finds the expression for the charge density:

$$
\rho_{\mathrm{sc} \rightarrow \mathrm{d}}=\frac{k T \epsilon}{z e}\left(\frac{2.405}{r_{0}}\right)^{2}
$$

At a charge density above $\boldsymbol{\rho}_{\mathrm{sc} \rightarrow \mathrm{d}}$, space-charge-driven ion loss should dominate diffusional loss whereas below that density, diffusional loss should dominate. It is noteworthy that (for singly charged ions) this switch occurs at a charge density that only depends on the temperature and the capillary radius. For the corona experiments in Figures 4 and 5, eq 13 gives $\rho_{\mathrm{sc} \rightarrow \mathrm{d}}=7$ $\times 10^{6}$ ions $/ \mathrm{cm}^{3}$. This corresponds to $95 \mathrm{pA}$ at $5 \mathrm{~L}$. $\mathrm{atm} / \mathrm{min}$. Indeed, the "SC" line in Figure 5 starts to diverge from the experimental data at about $100 \mathrm{pA}$. Clearly, in our experiments where the ion density at the inlet is about $2 \times 10^{8}$ ions $/ \mathrm{cm}^{3}$, space-chargedriven loss is quite important in the beginning of the transport capillary. It is interesting to note that for a 2.1-mm capillary, the potential difference between the capillary center and the periphery due to space- ${ }^{-}$charge at $3 \times 10^{8}$ ions $/ \mathrm{cm}^{3}$ is not large $(<1 \mathrm{~V})$. The capillaries used in electrospray-mass spectrometer interfaces are frequently narrower $(0.5 \mathrm{~mm})$. According to eq 13 , the switchover from space-charge-driven ion loss to diffusional loss occurs at a much higher charge density $\left(>1 \times 10^{8}\right.$ ions $\left./ \mathrm{cm}^{3}\right)$ in these narrow capillaries and diffusion thus should be the dominating ion loss process throughout the capillary.

An analytical theory for ion transport that combines space-charge effects and diffusion does not seem to exist. Here, we will assume that the total transmission efficiency in a segment of the capillary equals the product of the transmission efficiencies calculated separately for space-charge-driven and diffusional loss, respectively. Because these loss processes are of comparable magnitude only within a short capillary section, this should be a good approximation. A finitedifference method was then used to obtain the line marked "SC + Diff" in Figure 5. The gas throughput was $5 \mathrm{~L} \cdot \mathrm{atm} / \mathrm{min}$, the temperature was $308 \mathrm{~K}$, and the pressure was $1 \mathrm{~atm}$. The mobility constant used, $2.5 \mathrm{~cm}^{2} / \mathrm{V} \cdot \mathrm{s}$, was that for the expected $\mathrm{H}_{3} \mathrm{O}^{+}\left(\mathrm{H}_{2} \mathrm{O}\right)_{n}$ ions in ambient air. The corresponding diffusion constant, $0.064 \mathrm{~cm}^{2} / \mathrm{s}$, was obtained from $\mathrm{eq} 12$. (Thus, 
this calculation has no adjustable parameters.) Considering the assumptions involved, the agreement is better than could be expected. There is a significant deviation only for the 20 -fA point. It is not unlikely that this very small current measurement is in error. (It is also possible that aerosol particles already present in ion source air were charged by the corona discharge [21]; sec discussion below.) To fit the $7-\mathrm{L} \cdot \mathrm{atm} / \mathrm{min}$ data a higher mobility, and thus diffusion constant, had to be used. This discrepancy is likely due to an increased ion loss rate because of turbulent flow. We conclude that with regard to the transmission of corona ions through narrow capillaries, there is good agreement between experiments and calculations based on conventional ion loss processes and there is no need to search for more exotic explanations to explain a high ion transmission.

\section{Electrospray Results}

It was seen in the foregoing text that the capillary transmission efficiency of corona ions is well explained, within a factor of 2 , by the combined effects of space-charge-driven and diffusional ion losses to the capillary wall. We will now consider the possible reasons for the much higher transmission efficiency of electrospray ions, Figures 2, 3, and 5.

It was shown in Figure 3 that $I_{\text {trans }}$ for ES ions was still in the picoampere range for a 1-atm ion residence time of over $3 \mathrm{~s}$ in the 2.1-mm capillary. In contrast, the preceding "SC + Diff" calculation predicted a transmitted current many orders of magnitude lower. A possible explanation for this discrepancy is that, whereas the corona discharge produces only fairly small gas-phase ions, the electrospray in addition produces larger ions or charged particles that have slower diffusion and lower mobility. Next we will see how this assumption can be used to model the electrospray ion result in Figure 3 via the "SC + Diff" calculation. Presumably, the heavy ions or charged particles have a continuous distribution of diffusion constants. However, to simplify the calculation of $I_{\text {trans }}$ it was assumed that only three groups of ions, or charged particles, with three different diffusion constants (and corresponding mobility constants) were present. When small and large ions with different diffusion constants are present in the gas stream, they diffuse independently. However, their space-charge-induced drift toward the wall is determined by the total space-charge. Thus, the drift of small ions toward the wall will be faster because of the presence of the heavy ions.

The combinations of diffusion constants and abundances that gave the best least-squares fits to the electrospray and corona data in Figures 3 and 5, respectively, are shown in Table 1 . The calculated $I_{\text {trans }}$ for the electrospray is also plotted in Figure 9. The agreement with the experimental data is reasonably good. However, the calculated data show a much more pronounced increase in $I_{\text {trans }}$ with increasing gas
Table 1. Ion population distributions that give optimum fits to the transmitted ion current curves in Figures 3 and 5 , respectively.

\begin{tabular}{lcc}
\hline Electrospray & $\begin{array}{c}\text { Abundance } \\
(\%)\end{array}$ & $\begin{array}{c}\text { Diffusion } \\
\text { constant }^{\mathrm{b}}\left(\mathrm{cm}^{2} / \mathrm{s}\right)\end{array}$ \\
\hline \hline Electrospray, Figure 3 & 71 & 0.05 \\
& 27 & 0.008 \\
& 1.8 & 0.0016 \\
$\begin{array}{l}\text { Corona discharge. } \\
\text { Figure 5 }\end{array}$ & 100 & 0.08 \\
\hline
\end{tabular}

${ }^{a}$ It is likely that the actual distribution of the ES ions is more or less continuous.

${ }^{\mathrm{b}}$ The diffusion constants used in the calculation of diffusional loss were divided by 1.6 to correctly simulate the effect of the parabolic flow profile (see text).

throughput (at a constant $t_{\text {res }}^{0}$ ) than the experimental data. We believe that this is due to the neglect, in our model, of the effect of gas turbulence on ion loss. Thus, the model would tend to overestimate the ion transmission at high throughput. This introduces extra uncertainty in the abundances given in Table 1. In particular, this is the case for the relative abundance of low and intermediate mobility ions. Thus, the abundance of the intermediate mobility ions can be varied from about 20 to $90 \%$ while retaining fair agreement with experimental data. Indeed, the best fit to the low throughput data was obtained with high abundances of intermediate mobility ions. Similarly, the relative abundance of the low mobility charges could be from 1 to $4 \%$. Even though the model and the experiments do not give us exact abundances, the main conclusion is quite clear: When ions are produced by electrospray ionization, a large fraction of the transmitted current is carried by low mobility charges. The data in Table 1 were also used to calculate $I_{\text {trans }}$ for the experiments in

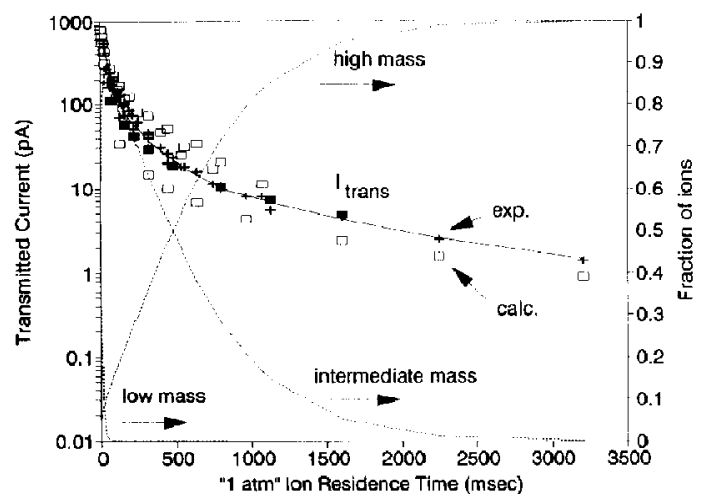

Figure 9. Comparison between the electrospray transmitted ion current $\left(I_{\text {trans }}\right)$ determined experimentally $(+)$ and calculated $(\square, \square)$, with the diffusion/space-charge model and the data in Table 1 . The gas throughputs in the calculations were $1,3,4$, and $5(\square)$ and $2(\square) \mathrm{L} \cdot \mathrm{atm} / \mathrm{min}$. The lines show the calculated relative transmitted current of low, intermediate, and high mass charge carriers. 
Figure $2 a$ and $b$, as shown by the solid lines in those figures. The agreement with experiment is good at a low gas throughput. However, at the highest gas throughput, the calculated $I_{\text {trans }}$ is too low or too high, respectively.

Mobilities of gas-phase ions at $25{ }^{\circ} \mathrm{C}$ and 1 atm range from 0.5 to $3 \mathrm{~cm}^{2} / \mathrm{V} \cdot \mathrm{s}$ [20]. By using Einstein's relation (eq 13) it is found that the diffusion constants range from 0.013 to $0.077 \mathrm{~cm}^{2} / \mathrm{s}$. As previously noted, a large fraction of the ions (charges) produced in electrospray are associated with significantly smaller diffusion constants, in the range of 0.001 to $0.005 \mathrm{~cm}^{2} / \mathrm{s}$. This is about 10 times lower than the diffusion constants found for ions with a mass of about $10^{3} \mathrm{u}[20]$. It seems, therefore, that this current must be carried by aerosol particles. What is the size range of such charged particles? The mobility of aerosol particles, for which the radius is large compared to the mean free path, can be calculated from Stoke's law [17]. For small particles, it is necessary to apply a correction factor to Stoke's law [17]. For example, one finds that a $50-\AA$ radius aerosol particle, with a mass of about $300,000 \mathrm{u}$ has a 1-atm diffusion constant of $5 \times 10^{-4} \mathrm{~cm}^{2} / \mathrm{s}$ [21]. By interpolating between the ion data $[20]$ and the aerosol data [21] it is found that the radii of the ES particles are in the $10-25-\AA$ range. We thus tentatively conclude that about $2 \%$ of the electrospray current is carried by particles that are predominantly within this size range. The mass of such particles should be on the order of 10,000-100,000 u.

Because no high molecular weight analytes were added in the ES experiment, the aerosol particles should be either small droplets that do not fully evaporate in the transport capillary or residual solid salt particles. At higher temperatures the droplets should quickly evaporate. As a result, the diffusion constants should increase and $I_{\text {trans }}$ should decrease. The opposite observation-that the transmitted current increases with increasing ion source temperature (Figure 7) - suggests that the particles are solid and that possibly their average size increases with increasing ion source temperature. Furthermore, the use of a dry nitrogen curtain gas in front of the transport capillary should ensure complete droplet evaporation and any residual current should be due to salt particles. In such experiments, no changes in transmitted current were observed. However, we are not confident that the curtain gas was totally effective in excluding room air from entering the transport capillary.

The observation that electrospray produces a significant flow of charged aerosol particles is interesting with regard to the problem of the mechanism of electrospray. It is noteworthy that fairly large multimers recently have been observed in electrospray $[22,23]$ and that their mechanism of formation is unclear. One could consider that the charged particles represent the extreme limit of large multimers. However, the solid particles observed here likely contain counterions and thus in our experiments would consist mainly of sodium acetate. If the electrolyte concentration in electrospray is above about $10^{-5} \mathrm{M}$, a significant fraction of the counterions are indeed ejected in the spray [24]. Because such ions are not observed in the mass spectra, they have to be present in larger droplets or particles. Thus, the aerosol particles observed in this work may be viewed as the inevitable result of the presence of counterions in the spray. Such particles would contribute to the electrospray current, but not to the electrospray ion signal.

The three lines in Figure 9 show the relative contributions from the low, intermediate, and high mass ions in Table 1 to the transmitted current, as a function of ion residence time $t_{r}^{0}$. The discrimination against low mass ions with large diffusion constants is very severe. The very rapid decay of the low mass ions is due to their faster diffusion. However, the presence of the heavier ions or particles also increases the space-charge. According to the calculations, this increases the loss of the low mass ions over what it otherwise would have been by a factor of 100 at $90 \mathrm{~ms}$ and a factor of 1000 at $160 \mathrm{~ms}$. For narrow and long capillaries, discrimination against low mass ions still could be severe, but is due mainly to differences in diffusion constants.

The loss of low mass ions in the transport capillary may be beneficial in biochemical mass spectrometry where high molecular weight ions are studied. On the other hand, it could be a severe disadvantage for the study of low molecular weight compounds. Mass discrimination also introduces considerable complications for the interpretation of kinetic experiments performed in capillaries [25]. Note, however, that the discrimination against low mass ions will be less severe in narrower capillaries, where space-charge effects are less important, and in shorter capillaries, where diffusional losses are less important.

\section{Glass Capillaries}

It has been noted already that the transmitted currents were very similar for glass and metal capillaries. We also have seen that it is critical to the successful treatment of ion transport in metal capillaries to assume that the ions are discharged as they hit the wall. Indeed, we measured a substantial wall current in metal capillaries. Despite the fact that in glass capillaries there is no wall current, $\boldsymbol{I}_{\text {trans }}$ still behaves as if ions were lost through diffusion (and space-chargeinduced ion drift). This is vividly demonstrated by the surprising result in Figure 8 that $I_{\text {trans }}$ was dependent on gas throughput but not on the capillary diameter. This can be understood as follows. When ions are lost by diffusion, the charge density is proportional to $\exp \left(-t / \tau_{1}\right)$; see eq $8 \mathrm{~b}$. Furthermore, the decay time $\tau_{1}$, is directly proportional to the square of the capillary radius (eq 8 a). However, at a fixed gas throughput and with a constant length capillary, the ion residence time $t$ is also proportional to the square of the capillary 
radius. For this reason, the charge density at the capillary exit and thus $I_{\text {trans }}$ should be independent of capillary radius at constant gas throughput. This is indeed what is observed; see Figure $8 \mathrm{~b}$.

It is strange that the transmitted current through glass capillaries should follow the diffusion model. Because there is no wall current, no ions presumably diffuse toward the wall! We have no clear evidence of what happens. On the other hand, we have found no alternative to the following argument. Assume that all ions that enter the capillary are positive. Some of the ions that first enter the capillary will hit the wall and charge the inner surface. However, except for the possible presence of a negligible wall creep current, once a steady state has been reached there will be no more net charging of the wall. It seems impossible that ions adsorbed on the wall could be re-ejected into the gas because the energy barrier is just too high (compared to thermal energies). We must therefore conclude that essentially no ions are hitting the wall after a steady state has been reached. Still, ions are moving through the capillary. The only possibility seems to be that there is a radial electric field at the capillary wall directed toward the center of the capillary. This would offset the diffusion of ions toward the wall. It is very important to note that such a (average) field cannot be created by a positive charge on the wall. This is a consequence of Gauss' law [26],

$$
\bar{\nabla} \cdot \bar{E}=\frac{\rho}{\epsilon}
$$

or

$$
\int_{\substack{\text { closed } \\ \text { surface }}} \bar{E} \cdot \bar{n} d a=\int_{\text {volume }} \rho d V
$$

where $\bar{E}$ is the electric field vector and $\bar{n}$ is a unit vector normal to the surface $a$. The integrated form of Gauss' law (eq 14b) states that the net flux of the electric field from a volume is proportional to the charge inside. This can be applied to an imagined cylinder positioned inside a long section of ion transport capillary such that the curved cylinder boundary is just inside the solid capillary wall. Because the space-charge inside such a cylinder is positive, the electric field at the cylinder boundary must be directed away from the center. It should be noted that the size of a uniformly distributed charge on the capillary wall does not affect the electric field inside the capillary; the ions will never drift toward the center! (This is strictly true only for the average electric field. If a positive charge is positioned just outside of the boundary of our imagined cylinder, the electric field in the boundary, close to the charge, is reversed.) Thus, ions are by necessity lost to the capillary wall. However, there is another possibility. First assume that space-charge is negligible. Then the (integrated) electric field flux from the cylinder discussed in the foregoing text is practi- cally zero (eq 14b). Now consider what happens if the axial electric field increases along the capillary. The electric flux into the imagined cylinder, through the front end, is now smaller than the flux out of the cylinder, through the back end. Thus, there must be an electric field flux into the cylinder at the cylinder wall. The direction of this electric field is that required to offset ion diffusion to the wall. It is possible that this process is self-regulating in that wall charging continues until it is just sufficient to generate the potential distribution required to make diffusional loss to the walls negligible. This might explain why diffusion still governs the transmitted ion current.

In the foregoing scenario, surface charging increases the potential at the capillary entrance, and this prevents most of the ions around the capillary entrance from entering into the capillary. Furthermore, the transmitted current is equal to the current entering the capillary.

"Ion tubes" or "ion funnels" with no ion loss can also be created by applying an external electric field distribution to a tube or capillary. A closely related subject is that of focusing of ions at atmospheric pressure [27]. Note, however, that this may not be a way to increase the sensitivity of high pressure ion sources. According to the unipolar formula (eq 3), the ion density will not increase along such an ion tube and thus the current sampled through an orifice may not change [14].

\section{Implications for Capillary Ion Source Interfaces}

Here we will discuss how to estimate the ion losses in capillaries that are used in actual electrospray interfaces. These capillaries typically have diameters from 0.2 to $0.5 \mathrm{~mm}$ and lengths from 10 to $20 \mathrm{~cm}$. The pressure on the outlet side of the capillary in such interfaces is always low (on the order of 1 torr) and this results in a gas throughput of about 10 torr $\cdot \mathrm{L} / \mathrm{s}$ $(0.8 \mathrm{~L} \cdot \mathrm{atm} / \mathrm{min})$. Comparable throughputs were used in the present study. However, our capillaries had diameters at or above $1 \mathrm{~mm}$ and the capillary outlet pressure was varied from a few torr to close to atmospheric pressure. Despite these differences, it is a reasonable assumption that the ion loss processes will be the same and that they will be determined by the same equations. The gas flow in the electrospray interface capillaries can be estimated by using Poiseuille's equation,

$$
Q=\left(\frac{\pi d^{4}}{128 \eta L}\right) P_{\text {ave }} \Delta P
$$

where $Q$ is the gas throughput, $\eta$ is the viscosity of the gas, $d$ is the inner diameter, $L$ is the length, $P_{\text {ave }}$ is the average pressure, and $\Delta P$ is the pressure drop across the capillary. Poiseuille's equation is strictly valid only for incompressible flow with no turbulence. 
However, for our own 0.5-mm-i.d. and 20-cm-long interface capillary, we calculated a maximum throughput of 14 torr $\mathrm{L} / \mathrm{s}$ from eq 15 . The measured value was only somewhat smaller -9.6 torr $\cdot \mathrm{L} / \mathrm{s}$. From the latter value, one calculates a Reynolds number (eq 9), of 2100. This means that the flow should be in the transition regime. For smaller capillaries of similar length, the flow becomes laminar and eq 15 should give an even better approximation of the gas throughput.

By combining eq 15 with eqs 2 and 8 , an expression for the ion transmission efficiency $T_{\text {eff }}$, can be derived:

$$
T_{\text {eff }} \approx b \exp \left(-\frac{1480 L^{2} D \eta}{d^{4} P_{\text {atm }}}\right)
$$

This equation can be used to give a rough estimate of the ion transmission efficiency through a capillary with length $L$ and inner diameter $d$ smaller than about 0.5 $\mathrm{mm}$. As before, $D$ is the ion diffusion constant. The constant $b$ incorporates the effects of enhanced ion loss due to higher diffusion modes, turbulent flow, and space-charge, and is therefore smaller than 1 . For narrow capillaries (i.d. $<0.5 \mathrm{~mm}$ ), a reasonable value for $b$ may be 0.5 .

As long as the absolute value of the expression within parentheses in eq 16 is smaller than 1 , the ion loss in the capillary is fairly small. For typical ions $\left(D=0.05 \mathrm{~cm}^{2} / \mathrm{s}\right)$ in air $\left(\eta=1.8 \times 10^{-4} \mathrm{P}\right)$ this condition is given by

$$
d>\left(1.315 \times 10^{-8} \mathrm{~cm}^{2} \times L^{2}\right)^{1 / 4}
$$

For example, for an $L=20-\mathrm{cm}$ capillary, eq 17 predicts that ion loss is serious only if the capillary diameter is below $0.5 \mathrm{~mm}$. However, with a diameter of 0.2 to 0.3 $\mathrm{mm}$, the predicted ion losses are very large. It must be stressed, however, that eqs 15 and 16 give order of magnitude estimates only. Also, transmission will be increased for charged droplets and aerosols.

\section{Appendix 1. Monte Carlo Simulations of Ion Diffusion in Capillary.}

Ion diffusion in the transport capillary was simulated by using a Monte-Carlo method. In these simulations, one ion was followed at a time. First, the ion was injected into the entrance of the transport tube. After injection, the ion was made to take a step of length $\Delta l$ in a plane perpendicular to the direction of the gas flow, at fixed time intervals $\Delta t$. The direction of the step in the plane was determined by a random number. The diffusion constant $D$ in the Monte Carlo simulation is related to $\Delta t$ and $\Delta l$ through [28]

$$
D=\frac{(\Delta l)^{2}}{4 \Delta t}
$$

The size of $\Delta t$ was chosen small enough that a good simulation of diffusion was achieved, but not so small that the calculation time became prohibitive. At every time step, the progress of the ion down the capillary tube was followed by letting the ion drift with the neutral gas flow. As the ion either exited the tube or hit the wall, its position was recorded.

The accuracy of the simulations was tested in a first series of calculations where all ions were injected into the exact center of an infinitely wide tube and where the gas flow velocity was constant over the cross section of the tube ("plug" flow). This is simply diffusion in two dimensions, and the radial ion number density at the tube exit is then given by

$$
n(r)=\frac{S}{(4 \pi D t)^{3 / 2}} \exp ^{\left(-\frac{r^{2}}{4 D t}\right)}
$$

where $S$ is a normalization constant [17] and $t$ is the time after injection. Figure 10 shows a comparison between the radial number density calculated from eq 19 and the density obtained in a Monte Carlo simulation with 30,000 ions. The agreement between the analytical expression (eq 19) and simulation is seen to be excellent.

The Monte Carlo program was next used to study the effect of the parabolic flow profile on the diffusional loss of ions. Here, the radial distance from the center to an injected ion was determined by a random number in such a way that a constant ion density in the gas entering the capillary was simulated. Furthermore, at each time step the local gas flow velocity was calculated from eq 10 . Figure 11 shows some results for an 2.1-rmm-i.d. capillary with a gas flow of 1.0

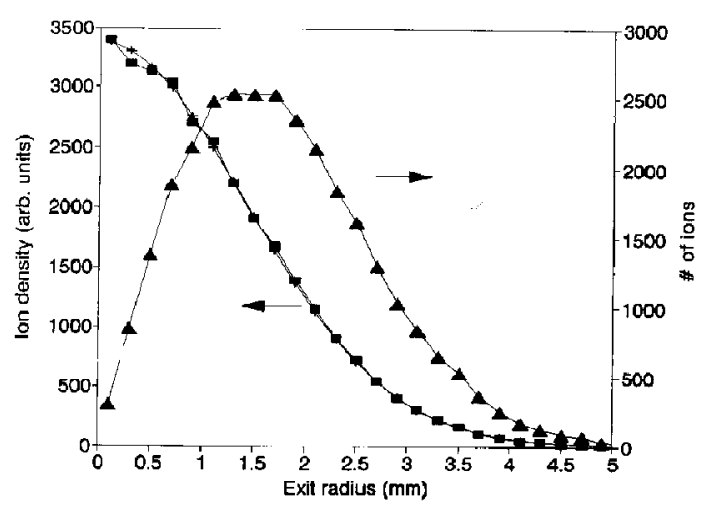

Figure 10. Monte Carlo simulation of the radial distribution of ions exiting an infinitely wide tube with a constant gas velocity and with a $0.2-s$ ion residence time. The ions were injected in the exact center of the tube. The curves show density $(\boldsymbol{E})$ and number of ions $(\Delta)$. The ion density distribution obtained from eq $19(+)$ is shown for comparison. The following parameters were used in the simulation: $\Delta l=0.1 \mathrm{~mm}, \Delta t=0.5 \mathrm{~ms}$, and $D=0.05 \mathrm{~cm}^{2} / \mathrm{s}$. The number of simulated ions was 30,000 and each ion was followed for 400 steps for a total ion residence time of $0.2 \mathrm{~s}$. 


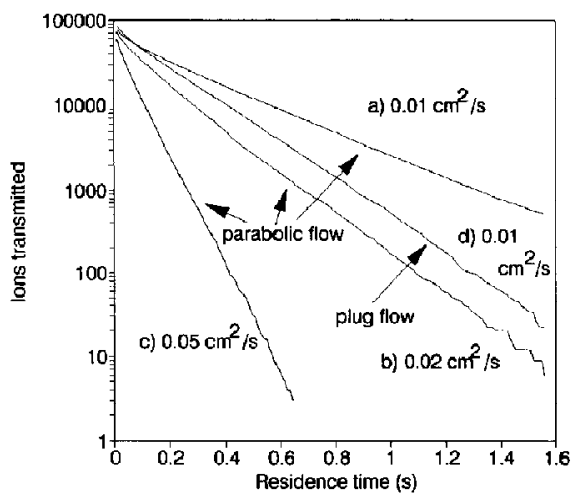

Figure 11. Monte Carlo results for the number of transmitted ions ( $\left.\alpha I_{\text {trans }}\right)$, with different diffusion constants, as a function of the 1 -atm ion residence time $\left(t_{\mathrm{res}}^{0}\right)$ in the transport capillary: $(\mathbf{a}-\mathbf{c})$ parabolic flow profile; (d) plug flow profile.

$\mathrm{L} \cdot \mathrm{atm} / \mathrm{min}$. Curves $\mathrm{a}, \mathrm{b}$, and $\mathrm{c}$ show the number of transmitted ions for a parabolic flow profile (with different diffusion constants). For comparison, curve d shows a result for a plug flow profile. In all simulations $10^{5}$ ions were injected. The initial fast decrease in the ion signal is due to the rapid decay of the higher diffusion modes (enhanced loss of ions injected close to the wall). However, after the (flow-modified) fundamental diffusion mode has been established, the transmitted current decreases exponentially with capillary length, as shown by the straight lines in the semilogarithmic graph.

A comparison between curves a and $d$ in Figure 11 shows that the ion loss is much smaller with parabolic flow than with plug flow for the same diffusion constant. However, both curves show exponential decays. The decay time for "plug" flow (curve d) is $0.137 \mathrm{~s}$, which is in excellent agreement with the $0.132 \mathrm{~s} \mathrm{calcu}$ lated from eq 8 . In contrast, the decay time for the parabolic flow profile is $0,224 \mathrm{~s}$. Thus, the effect of the parabolic flow is equivalent to increasing the half-life of the ions in the capillary by a factor of $\sim 1.6$. Alternatively, one may consider that the effective diffusion constant has decreased by the same factor. For a long capillary where ions may spend many half-lives, this causes a dramatic increase in the transmitted current.

\section{Acknowledgment}

The authors thank Reed Howald for help with and the loan of the Montana Interface.

\section{References}

1. Yamashita, M.; Fenn, J. B. J. Chem. Phys. 1984, 88, 4451.

2. Fenn, J. B.; Mann, M.; Meng, C. K.; Wong, S.F.; Whitehouse, C. M. Science 1989, 246, 64 .

3. Bruins, A. P. Mass Spectront. Rev. 1991, 10, 53.

4. Horning, E. C.; Horning, M. G.; Carroll, D. L.; Dzidic, I.; Stillwell, R. N. Anal. Chem. 1973, 45, 936.

5. Reid, N. M.; Buckley, J. A.; French, J. B.; Poon, C. C. Adv. Mass Spectrom. 1979, 8b, 1843.

6. Anderson, J. B.; $\Lambda$ ndres, R. P.; Fenn, J. B. In Advances in Atomic and Molecular Physics; Bates, D. R.; Estermann, I., Eds.; Academic: New York, 1965.

7. Whitehouse, C. M.; Dreyer, R. N.; Yamashita, M.; Fenn, I. B. Anal. Chem. 1985, 57, 675.

8. Shahin, M. M. I. Chem. Phys. 1966, 45, 2600.

9. Kambara, H.; Kanomata, I. Mass Spectrose. 1976, 24, 229.

10. Chowdhury, S. K.; Katta, V.; Chait, B. T. Rapid Commun. Mass Spectrom. 1990, 4, 81 .

11. Rockwood, A. L.; Busman, M.; Udseth, H. R.; Smith, R. D. Rapid Commun. Mass Spectrom. 1991, 5, 582.

12. Stearns, R. G. J. Appl. Phys. 1989, 66, 2253.

13. Carroll, D. I.; Dzidic, I.; Horning. E. C.; Stillwell, R. N. Appi Spec. Rer. 1981, 17, 337.

14. Busman, M.; Sunner, J.; Vogel, C. R. J. Am. Soc. Mass Spectrom. 1991, 2, 1 .

15. Sigmond, R. S. In Electrical Breakdown of Gases; Meek, J. M.; Craggs, J. D., Eds.; Wiley: Chichester, 1978; p 319.

16. Sigmond, R. S. J. Appl. Phys. 1982, 53, 891.

17. Mason, E. A.; McDaniel, E. W. Transport Properties of lons in Gases; Wiley: New York, 1988

18. McCabe, W. L.; Smith, J. C.; Harricott, P. Linit Operations of Chemical Enginecring; 4th ed.; McGraw-Hill: New York, 1985.

19. Smith, D.; Adams, N. G. Adv. At. Mol. Phys. 1988, 24, 1.

20. Shumate, C.; Louis, R. H. St.; Hill, H. H. Jr. J. Chromatogr. 1986, 373, 141.

21. Hinds, W. C. Aerosol Technology. Properties, Behavior and Measurement of Airborne Particles; Wiley: New York, 1982.

22. Winger, B. E.; Lightwahl, K. J.; Lou, R. R. O.; Udseth, H. R.; Smith, R. D. J. Am. Soc. Mass Spectrom. 1993, 4, 536.

23. Busman, M.; Knapp, D. R.; Schey, K. L. Rapid Commun. Mass Spectrom. 1994, 8, 211.

24. Ikonomou, M. G.; Blades, A. T.; Kebarle, P. Anal. Chem. 1990, $62,957$.

25. Winger, B. E.; Lightwahl, K. J.; Smith, R. D. I. Am. Soc. Mass Spectrom. 1992, 3, 624

26. Feynman, R. P.; Leighton, R. B.; Sands, M. The Feynman Lectures on Physics; Addison-Wesley: Reading, MA, 1964.

27. Potjewyd, J. Focussing of lons in Atmospheric Pressure Gases Using Electrostatic Fields, Ph.D. thesis, University of Toronto, 1983.

28. Berry, R. S.; Rice, S. A.; Ross, J. Physical Chemistry; Wiley: New York, 1980. 